\title{
The Faculty Evaluation Process: The First Step In Fostering Professional Development In An Online University
}

Barbara Weschke, Walden University, USA

Stephen Canipe, Walden University, USA

\begin{abstract}
The presentation will trace the experiences of two program directors from Walden University and their examination of issues involved with faculty assessment. Their experiences were used to create a simple but effective and workable model for faculty assessment and development. Once a presentation of how data were obtained, and analyzed, to create an effective communication process, the specifics of how faculty members were engaged in the collegial methods to ensure a workable professional development process will be described. The experiences of the directors in working with assessment will be utilized as they demonstrate how they regularly conduct an interactive evaluation process, involving the audience in using the model created at Walden in the Richard W. Riley College of Education and Leadership. This model was developed using various metrics, such as student course evaluations, faculty self-assessments, checklists of activity, adherence to rubrics, etc. The entire process begins with the premise that collegial interactions will provide the most sustainable changes. The process concludes with the implementation of online professional development in response to the collected data.
\end{abstract}

Keywords: faculty assessment, professional development; collegial interaction; higher education; online university.

\section{INTRODUCTION}

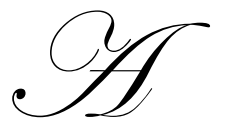

$\mathrm{s}$ in any educational institution of higher learning, evaluations of the university faculty's performance are critical measures, both of professional progress, and student achievement. In the distance-learning, online environment, the equivalent of an administrator entering the brick-andmortar classroom to observe an instructor occurs when one logs into the classroom; navigates its course-page areas; and assesses performance. Whether campus-based or online education, the reason for faculty evaluation basically is the same - to encourage the best performance and maximize student achievement.

\section{THE WALDEN UNIVERSITY ASSESSMENT MODEL: A HISTORY}

At Walden University, distance learning has been fostered nearly from its beginnings, in 1970. Each school within the university has some culture dealing with assessment. At Walden, the Total Quality Management (TQM) precepts guide the assessment functions in the Master's in Education (MSEd) program. Recognizing that performance and improvement of performance are goals to attain, the Walden faculty, in conjunction with administration, has used an instrument developed by the Office of Institutional Research and Assessment to collect student input. This is only one aspect of the metric. Other components include self-evaluations/assessments and drop-in visits into the classrooms. All of these are components of the culture of assessment, which has developed at Walden. As with any culture, the process was not linear, and was not imposed from above. It has grown from faculty recognition that the academic world exists in an assessment paradigm. This is certainly true of our students (practicing teachers who are seeking their master's degree) in their work environment. 
Walden's courses are also full of assessments; therefore, for all parties, theory and practice merge. Some class assessments, as well as faculty assessments, were undertaken to demonstrate to the Higher Learning Commission that the Walden courses, students, and faculty attain the standards required.

Beginning in 2007, Walden joined with other institutions in an assessment pilot, conducted by the Higher Learning Commission in the North Central Association; however, even before this focus, Walden faculty and staff were working on assessment issues. According to Canipe in The Department Chair, "The information [from course evaluations] is used for faculty assessment and development. It is available within a week of student completion of any course" (p. 16, 2006).

The Office of Institutional Research and Assessment produces research documents for faculty and administration, which focus on the attainment of the desired outcomes. The Learning Outcome Reports (LOR) was the first formal attempt in the MSEd program to quantify student achievement across the program.

Following shortly on the heels of student outcomes came the recognition that faculty improvement was a key component in improving student success. The specific process has evolved from yearly faculty evaluation cycles to a more continuous process, which includes quarterly Instructional Quality Reports (IQR). The IQR is compiled by the Office of Institutional Research and Assessment and then shared with administrators.

These quantifiable data result from student course evaluations. One part of course evaluation asks questions concerning faculty performance. This has been further broken down into a feedback substrate. This division and analysis came about during data analysis where it became obvious that the higher quality and higher frequency of faculty feedback caused student achievement to increase. Feedback has three components-1) substantive, 2) individualized, and 3) timely.

\section{THE MASTER'S IN EDUCATION FORMATIVE ASSESSMENT MODEL}

Administrators were acutely aware that reliance solely on student course evaluations could skew reality. With this in mind, other metrics were added, including drop-in classroom visits where the program directors or other administrators used a checklist, and checked the timeliness of response. Each faculty fills out a self-evaluation form, which asks, among other items, for issues, concerns, and problems. All of these metrics and processes were designed to provide a 360-degree view so that a comprehensive picture of the faculty member's accomplishments and areas for improvement could be obtained.

No amount of visiting or assessment of results can create a faculty group who is desirous of improvement. This is a self-initiated process that at Walden is encouraged through collegial conversations. Program administrators attempt to be assistant problem-solvers rather than a cudgel bearer to "beat" faculty into compliance. The achievement of results is due to faculty internalizing the assessment data and desiring and seeking ways to improve classroom performance.

As noted earlier, there is a culture of quality improvement that pervades the master's faculty group in the Richard W. Riley College of Education and Leadership at Walden University. The authors opine that the success in achieving faculty performance improvement comes from two factors: 1) experienced and quality faculty; and 2) administrative commitment to allow problem solving to enhance improvement.

To this end, a checklist was developed with faculty input and information about the following items, among others, and the data are reported to the instructor, and administrator:

- Determining whether posting the following documents in the classroom space occurs:

- Course Policies including a Late Policy

- Walden's official documents

- Application, Reflection, and Discussion Board Rubrics

$\circ$ Weekly announcement 
- Determining faculty presence, grading, and participation in the classroom according to the established guidelines (qualitative and qualitative assessment)

- Questions and Answers

- Student Lounge

- Discussion Board

- Application Assignments

Among the formative criteria an administrator may use to evaluate the online instructor's classroom performance are the following:

1. Discussion Board: Does the instructor respond to students on a regular basis, and with substantive comments?

2. Participation: Does the instructor regularly participate throughout the course, and interact appropriately with students?

3. Document sharing: Are required course, department, and university policies and documents visibly posted? Are faculty and students posting shared information?

4. Time spent (user activity): Does the instructor devote between 10-15 hours weekly?

5. Weekly announcements: Does the instructor provide weekly updates, announcements, and information for students' use?

6. Responses to questions: Does the instructor answer students' questions in a timely, effective manner?

7. Feedback: Is instructor feedback on students' papers timely, substantive, and individualized?

Evaluators use these criteria to communicate the results of classroom observational visits, and such visits are considered to be informal, though formative, evaluations; moreover, an instructor may expect to receive such a completed form at least twice each semester, along with follow-up email messages, and/or telephone calls, to discuss the results.

As a culture committed to using data-based decision making, the good use of data is key. Questions dealing with student perception are placed on the course evaluation and form a part of the larger faculty assessment process. These evaluation data are collected at the end of each course and over time give a good view of faculty performance and improvement. As an example the data from a recent course evaluation set is presented below.

As might be expected, these data, over time, provide a longitudinal view very helpful in determining the types of faculty development activities necessary. In Figure 1, it can be seen that question 3, 5, and 6 are lower than the others in this survey administration. These particular questions deal with encouraging independent thinking in discussions; responding to emails within the designated timeframe; and feedback showing how to improve one's work.

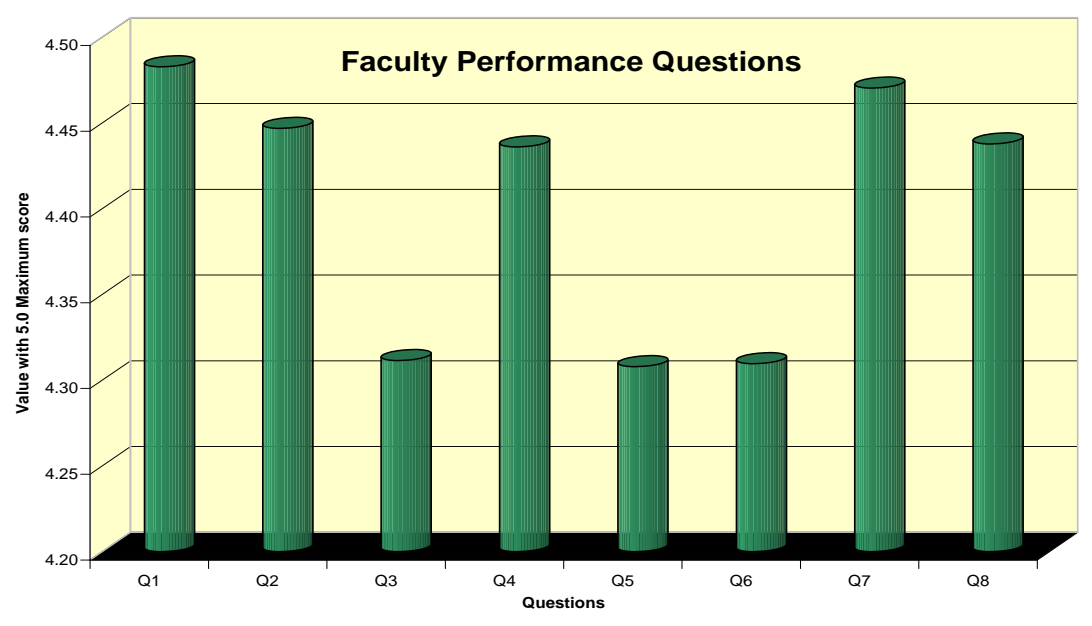

Figure 1. Faculty Performance Questions 
While these data are available for every instructor, and the program directors do address specific instances with faculty members, the data presented in this graph are for the entire several-hundred faculty teaching master's level courses in Walden University's Richard Riley College of Education and Leadership. In order to see whether these data are indicative of department-wide issues, more statistical work was done. It is necessary to determine reasons for email responses being delayed-in this case this was during a period of weather disruptions in several sections of the country causing faculty to have limited and/or intermittent access to the Internet and email. The other two areas where scores were lower than desirable - independent thinking and feedback - take a bit more analysis. But these are the issues that are examined for each faculty member and in the roll-up for the entire cadre of faculty at the end of each course.

Walden's Learning Management System (LMS) allows pulling either individual or group data as desired. Having the ability to reconfigure data sets yields a quicker response to any specific issues seen in the data. Moving forward this will mean a response targeted to issues identified by data and modifications occurring based on data that are near real-time.

\section{ENCOURAGING PROFESSIONAL DEVELOPMENT IN AN ONLINE ENVIRONMENT}

The data demonstrating faculty's effectiveness, or need for more effective faculty performance, are the not the source of professional development; they are the foundation on which communication with instructors is built. It must be emphasized again that these evaluations are not summative, rather they are formative; moreover, they are not intended to replace the university's more detailed, complete evaluation, usually provided to faculty on a onceyearly basis. These formative, informal evaluations are used as information on which to build the summative faculty evaluations. As stated before, faculty must be aware that their administrators monitor their classrooms for evaluative purposes. These visits may take place as pre-arranged observations, or "drop-in" visits, and may be informal, formal, formative, and/or summative in nature. Most of Walden's faculty members report they find the results useful in their practice. In most cases, the administrator informs instructors of the visits' results by way of a follow-up telephone conversation, to review the written evaluation together. In particular, faculty report they appreciate any constructive comments regarding their feedback on students' papers, and usually are receptive to learn better practices to communicate with students.

While the types of observations may vary, as may the reasons, communication with faculty on their progress significantly is critical to students' success, and satisfaction. At Walden University, most courses are lockstep, and one requirement is for certain documents, and policies, to be posted visibly in the online classroom; therefore, maintaining quality, and information for students' use, are a minimum for an instructor's performance during a formative assessment.

If the instructor is found to require assistance, additional training, and/or support, such as faculty development, must be considered an integral, ongoing element of her or his employment. In some cases, an instructor may inadvertently omit an element, and the formative evaluation is a reminder of this need. In others, patterns of ineffective practices may be uncovered, and remediated. As a result, such an evaluation is constructive for the instructor, meant to inform, rather than to chide. Finally, as a potential scenario, these observations, Professional Improvement Plans, and follow-up visits could result in a dossier for continued improvement, or potential termination, if the instructor does not improve performance.

According to Fang (2007), any evaluation of faculty must be constructive, and should result in improvement; moreover, the evaluator and instructor must be able to identify the reason or source of the missing component, especially if the instructor requires more training or skill building. This is the reason a constant, consistent communication among instructors, leaders, and students, is an effective measure of progress. It is these results that essentially form the basis of any type of faculty development, and must be regarded as developmental, not punitive.

This is a reason students' evaluations, along with administrators' monitoring classrooms, are significant. At Walden, students have opportunities to rate their instructors' performance through the questions, as mentioned above; however, students also are provided opportunities to write in their comments, and these comments carry a 
good deal of importance when evaluating faculty. However, if administrators carefully and consistently monitor classrooms, such patterns become apparent, long before students' comments reflect such breaches.

For formative, as well as summative faculty-performance assessments, it also is significant that administrators establish, and maintain, a visible presence, and be available to support instructors, which can engender continued motivation to conduct classes effectively; moreover, administrators must inform online instructors of the guidelines, rules, and parameters for performance evaluation, all of which should be transparent, and consistent among a university's programs.

For the online environment, therefore, a university may provide ongoing faculty development for all instructors' edification; however, for instructors who require more specific development, a Performance Improvement Plan (PIP), a collaborative plan, formed by the administrator and instructor, may be a formative, proactive choice to provide support, and goals for the instructor. The PIP is a more formal document, one that is placed in the instructor's personnel file, and updated throughout the duration of the instructor's professional development. Figure 2 is an example of the PIP form:

\section{Walden University College of Education Instructional Quality Co urse Ranking Feedback}

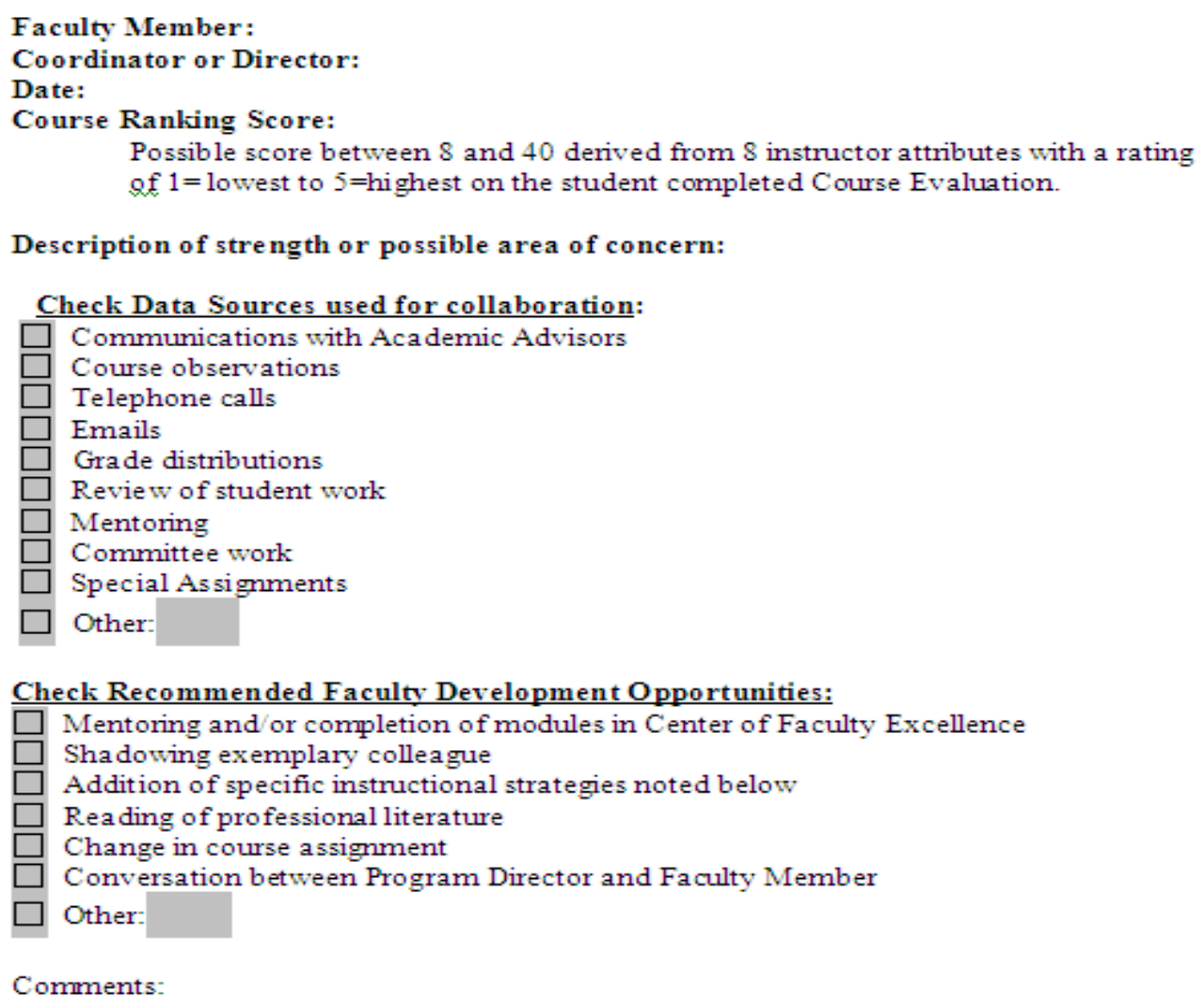

Figure 2. Faculty Performance Improvement Plan Form 
For instructors whose performance reviews continually reveal strengths, they may be rewarded by assuming more duties such as becoming course leads, coordinators, or program directors. In addition, these successful instructors may be assigned additional courses; assume a leadership role; and provide mentoring by example to their colleagues. Providing recognition for high-performing faculty might be in the form of certificates for high performance, an honorarium, or a more formal recognition, such as a university's achievement award. Figure 3 is an example of such opportunities afforded high-performing instructors.

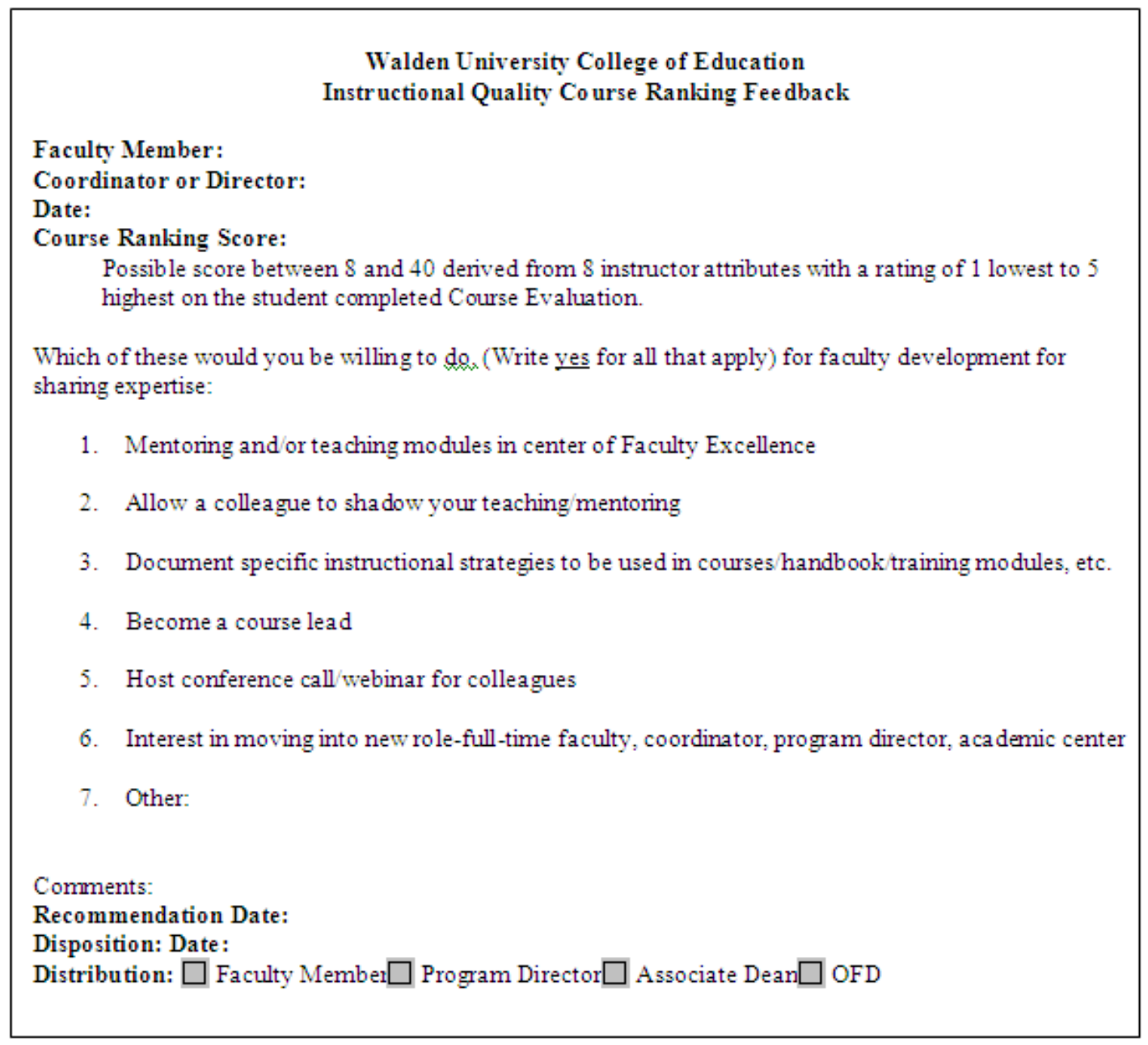

Figure 3. Opportunities Afforded High Performers

\section{THE MATTER OF BIAS AND SUBJECTIVITY IN EVALUATING INSTRUCTORS' PERFORMANCE.}

Elmore (2008) contends:

Because humans are social animals who form alliances, make friendships, and develop biases, the evaluation process is often subjective and may be affected by extrinsic factors. Indeed, there are many junctures in the steps leading to recognition or reward where individual administrative perception and bias can affect an evaluation. The subjectivity of established evaluation practices may lead some faculty members to conclude that their evaluators are unfair and the process is flawed. If many faculty members feel this way, discontent, erosion of morale, and a decline in the quality of the work environment will result. (38) 
This is one reason that the formative evaluations are objective, focusing on the recommended items, and practices in the online classroom. The intent here is to evaluate in a non-biased manner; moreover, the better an administrator knows the faculty, the more objective the evaluation. In conjunction with students' evaluations, which, in some cases tend to reflect personal biases, the formative checklist serves as a balance between them.

The bottom line is that an administrator must develop, and maintain, a constant, consistent pattern of communication with every instructor under her or his aegis. In the online environment, this may be accomplished through email, teleconferences, informal course-based chat rooms, and face-to-face conversations. The latter may occur during commencement, and university-wide regional meetings.

\section{WHAT THE FACULTY SAY}

To gather instructors' perspectives regarding the communication, the writers solicited feedback from the master's faculty.

Overall, faculty response indicated their satisfaction with assessment practices, as well as venues for their professional development. The results of the survey are presented in Figures 4-12 on the following pages, and the authors are grateful to all instructors who participated in this survey. Each figure is identified and an explanation is provided.

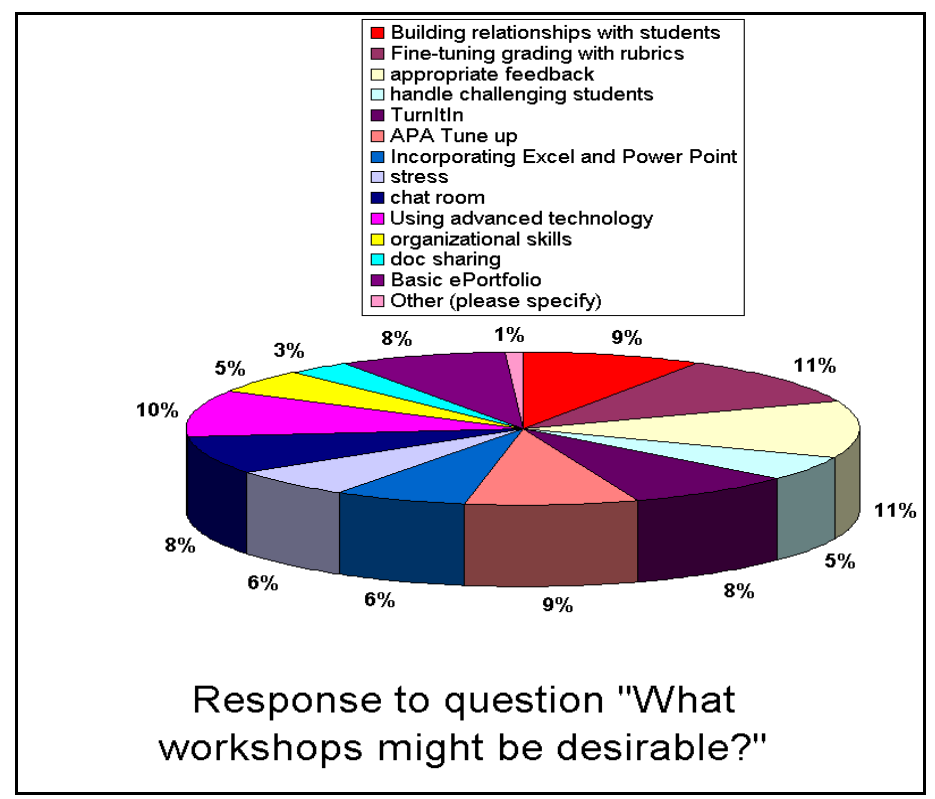

Figure 4 Initial Faculty Survey Data

During an initial survey, faculty was asked what types of staff development workshops might be desirable. The responses are shown in Figure 4. The $\boldsymbol{n}$ for this survey was 66.

Based on these responses, a series of workshops/self-training activities were created by faculty for faculty. After the initial survey, a follow-up survey was conducted to determine both the level of participation in the developmental workshops created by faculty for faculty and also the perceptions of faculty concerning evaluations and communications.

The data presented in the following Figures 5-12 show the responses. Figures 5-8 illustrate the demographics of the sample. And the subsequent ones (Figures 9-12) illustrate the various questions and responses. $\mathrm{n}=72$. 


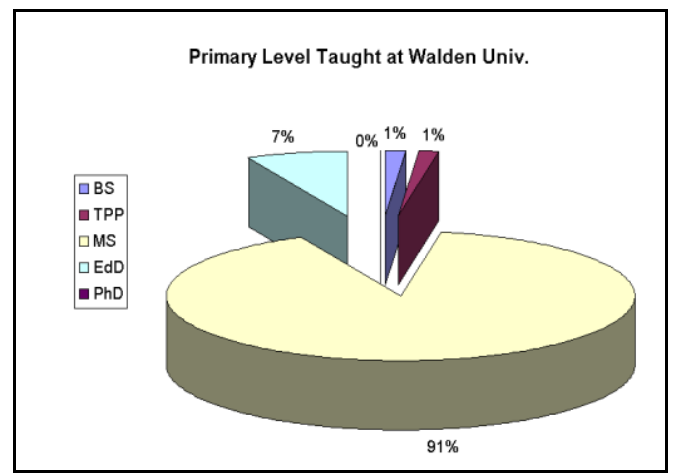

Figure 5. Primary Program Level Taught at Walden.

Figure 5 shows the demographics of those faculty members completing the follow-up survey on evaluation and faculty development.

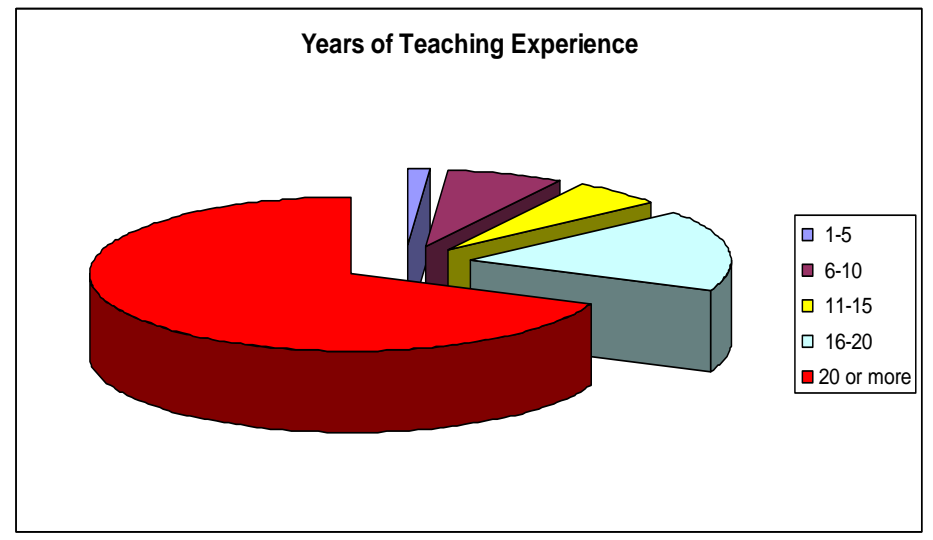

Figure 6. Number of Total Years of Faculty Teaching Experiences (all places)

The primary level taught at Walden is displayed in Figure 6. The $\mathrm{PhD}$ area is the one showing $0 \%$ respondents. This figure is indicating all years of teaching experience - not just at Walden University.

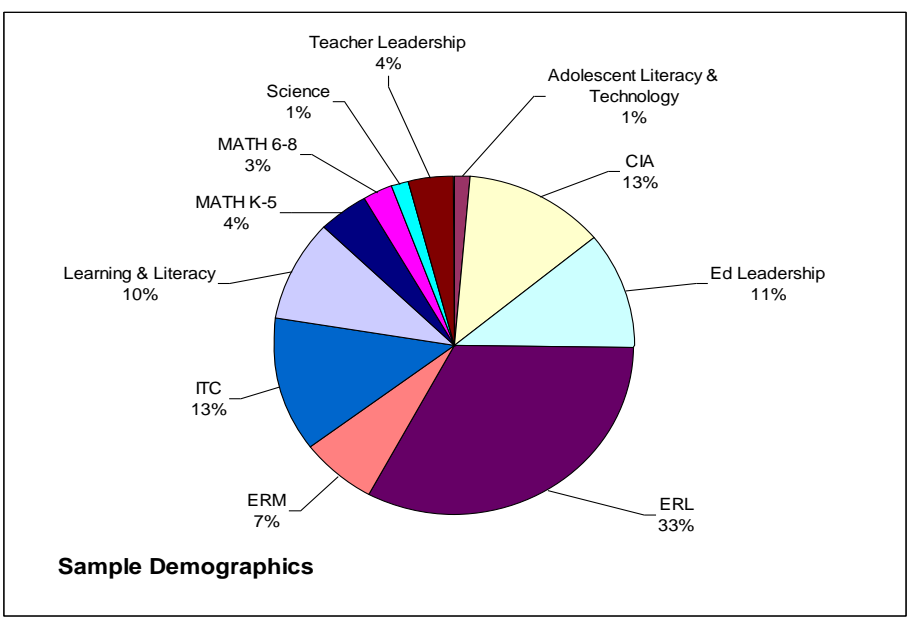

Figure 7. Respondents' Specializations 
The breakdown in Figure 7 shows areas taught for the $91 \%$ of respondents who currently teach in the MSEd programs. Not included in these data are the EdD, BS, or TPP areas.

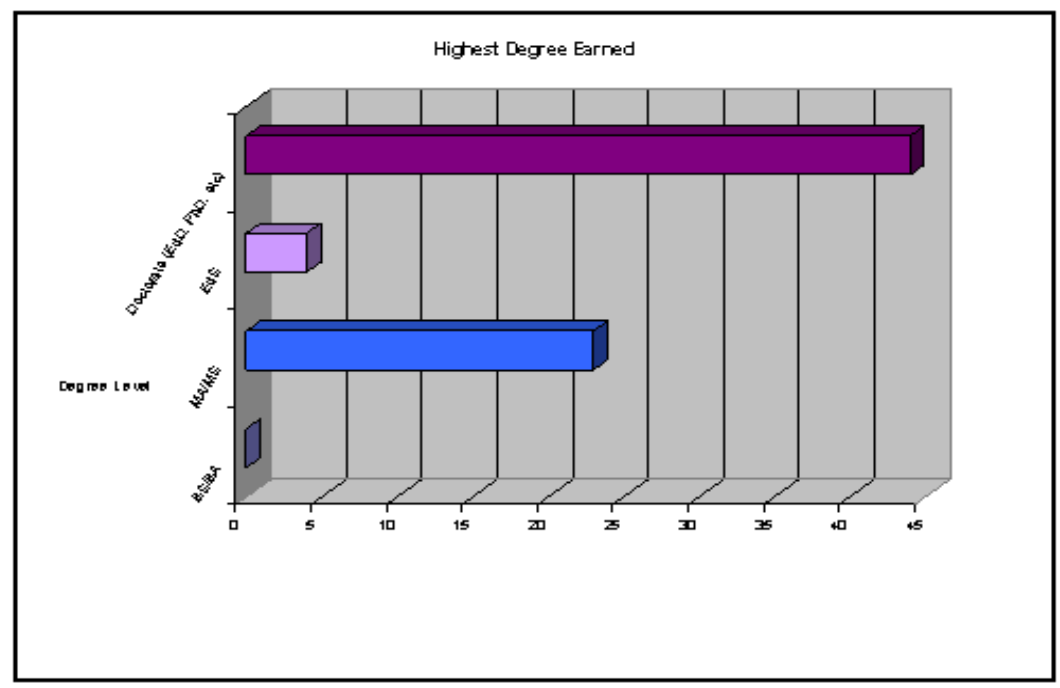

Figure 8. Highest Earned Degree by Faculty

Figure 8 reflects the highest degrees earned by the teaching faculty. The scale numbers on the bottom reflect actual numbers of respondents NOT percentages.

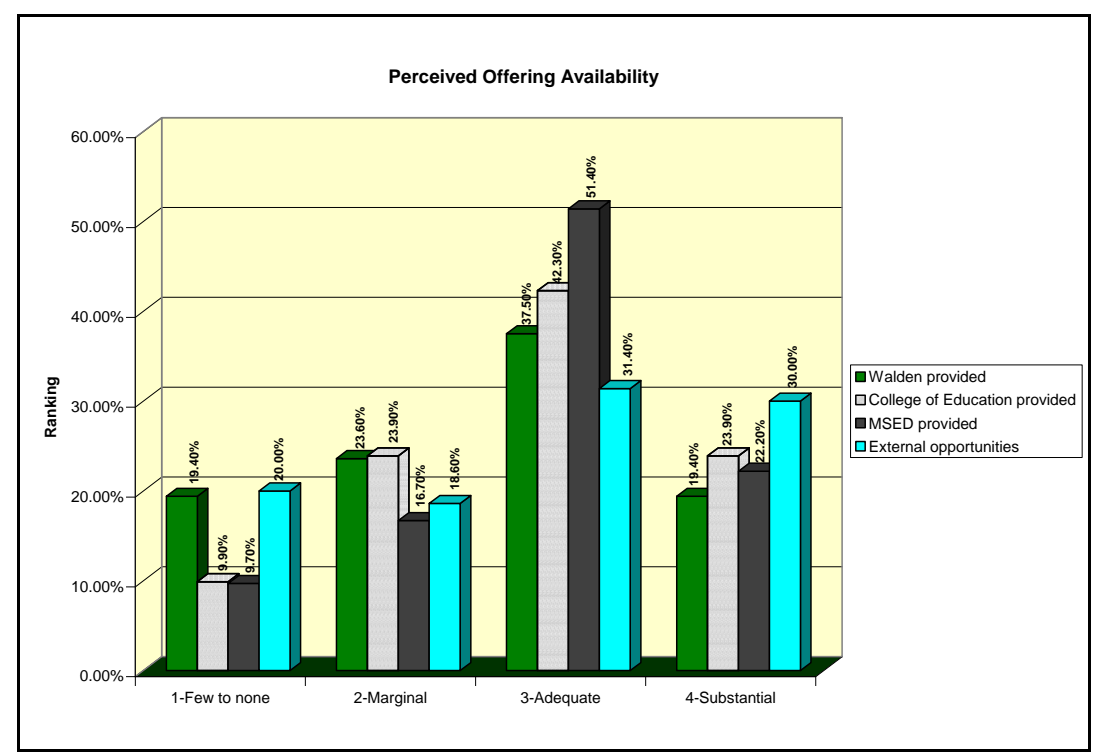

Figure 9. Faculty Perception of Availability of Staff Development

Faculty members were asked to rate the availability of staff development from various sources University, CoEL*, MSEd, outside according to a four-point Likert scale - Few to Substantial. The graphic in Figure 9 shows the reported perceptions. $(*$ CoEL $=$ College of Education and Leadership sometime referred to as RWRCoEL for the Richard W. Riley College of Education and Leadership) 


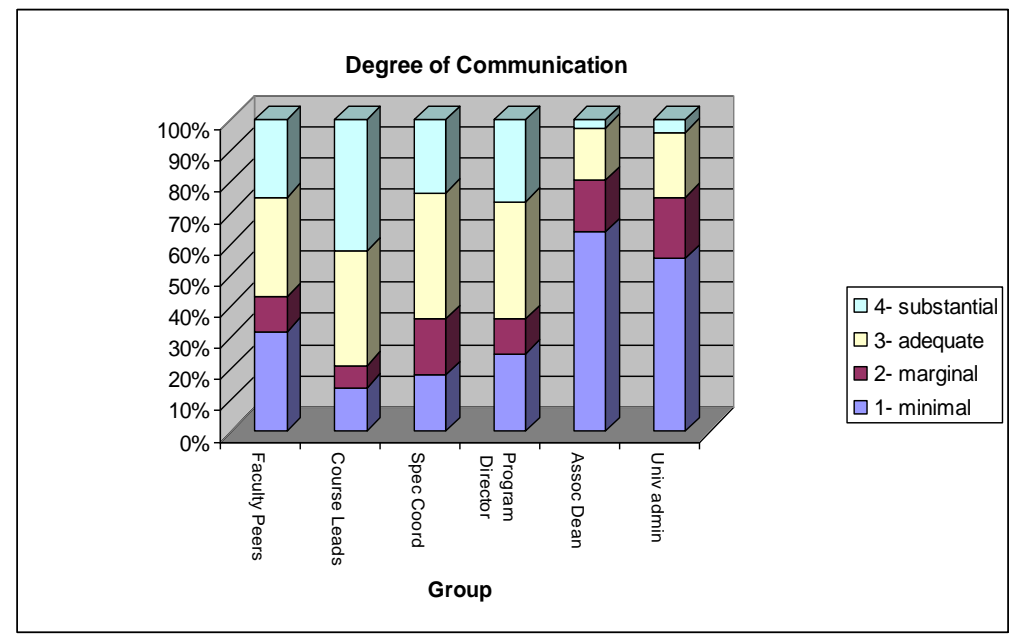

Fgure 10. Faculty Rating of Degree of Communication from Various Sources

Faculty members also were asked to respond to a question dealing with the degrees of communication/ interaction with various audiences from peers to the University administration. The scale was Likert-like - from minimal to substantial. The graphic in Figure 10 illustrates their responses.

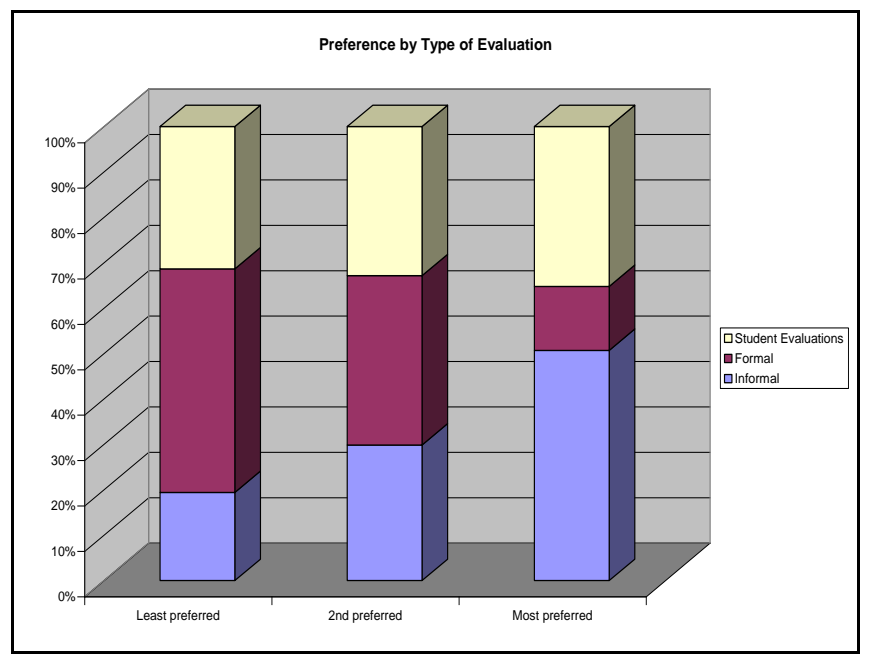

Figure 11. Faculty Ranking of Preferred Communication Methods

Faculty members were asked to state their preferences for three different types of evaluations - student, informal, and informal... and rank them on a least preferred to most preferred scale. The results are displayed in Figure 11. 


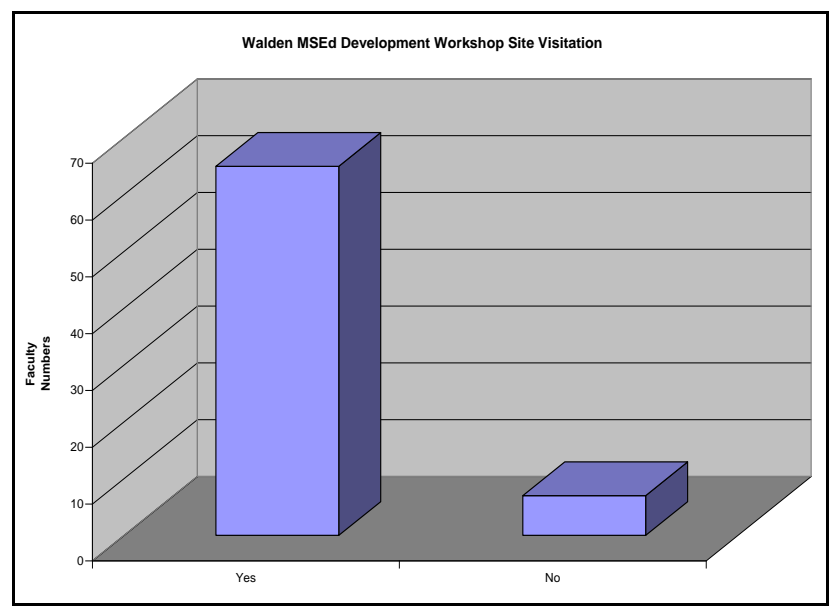

Figure 12. Faculty Report of Visits to the Staff Development Website

Faculty members were asked whether they had visited the development website on the faculty portal. The numbers of the respondents reporting visits was large with $90 \%$ reporting visiting $(n=65)$ while only $7(10 \%)$ reported not having visited the site. Figure 12 displays this information graphically.

Clearly, there is a culture of quality improvement that pervades the master's faculty group in the Richard W. Riley College of Education and Leadership at Walden University. The authors opine that the success in achieving faculty performance improvement comes from two factors: 1) experienced and quality faculty; and 2) administrative commitment to allow problem solving to enhance improvement.

\section{ENCOURAGING PROFESSIONAL DEVELOPMENT IN AN ONLINE ENVIRONMENT}

The data demonstrating faculty's effectiveness, or need for more effective faculty performance, are not the source of professional development; they are the foundation on which communication with instructors is built; moreover, while the types of observations may vary, as may the reasons, communication with faculty on their progress significantly is critical to students' success, and satisfaction. According to Fang (2007), any evaluation of faculty must be constructive, and should result in improvement; moreover, the evaluator and instructor must be able to identify the reason or source of the missing component, especially if the instructor requires more training or skill building. This is the reason a constant, consistent communication among instructors, leaders, and students, is an effective measure of progress. In the online environment, this may be accomplished through email, teleconferences, video feeds, informal course-based chat rooms, and face-to-face conversations.

\section{THE COURSE-LEAD MODEL}

The course-lead model allows Walden faculty members to communicate frequently with their leadership, and interact on a regular basis, formally at least twice a term, and informally, potentially every day.

Koops and Winsor (2005) maintain that a model such as this university's course-lead model, "is to encourage collaboration and conversations about the art and craft of teaching" (p. 68). In this manner, the peer-topeer interaction fosters a collegial sharing or strategies, and best practices in the online classroom, where coming from a higher-level administrator could be perceived as the instructor having done something "wrong." In this case, the peer interaction is better, and more correctly, perceived as collegial professional development. Moreover, using the course-lead model, these faculty members, who communicate frequently with administrators, and whom Walden University employs, interact on a regular basis, formally at least twice a term, and informally, potentially every day. 
Furthermore, faculty members share with one another their best practices, as well as keep up to date on course requirements and changes. As a result, these alliances to which Elmore (2008) refers, become collegial in nature, and the administrators, who frequently participate in the course-leads' conference calls, are provided with a well-rounded perspective of each instructor. It is through faculty development, based on the results of classroom observations, and students' evaluations, that online instructors continue to sharpen their teaching skills, thereby contributing to the community of scholar-practitioners the university fosters. Whether it is honing instructors' skills with APA formatting; developing research-based practices; tips for providing timely, substantive, individualized feedback on students' papers; or creating a safe classroom atmosphere, faculty members continue to share their expertise, experiences, and skills with one another, in a constructive, productive manner, based on performance evaluations' parameters. Therefore, as data presented here demonstrate, frequent, ongoing communication; an open forum; and a network for assessing, and developing faculty's performance are the keys to encouraging faculty development, in an active, collegial, responsive manner.

\section{THE ROLE OF PROFESSIONAL DEVELOPMENT AS A REMEDIAL EXERCISE}

For faculty whose online work requires assistance, Walden provides interactive, online facultydevelopment workshops through the university's Center For Faculty Excellence. In this venue, all faculty may gain access to these workshops; however, for instructors whose Professional Improvement Plan (PIP) requires additional skill building, administrators may require instructors to participate in the training, along with follow-up assessments and/or discussions of their effectiveness. Among these workshops are the following: Providing Students with Appropriate Feedback; Grading Students' Papers Using Rubrics; APA Guidelines for Scholarly Work; and How to De-Stress While Teaching Online. Each of these workshops has been developed by faculty committees, based on recommendations from their colleagues' recommendations from a survey sent to all instructors in the master's programs.

It is through faculty development, based on the results of classroom observations, and students' evaluations, that online instructors continue to sharpen their teaching skills, thereby contributing to the community of scholar-practitioners the university fosters. Faculty may avail themselves of the workshops twenty-four hours, seven days a week, and even may share these with their students. However, faculty development is not limited to the workshops; it continues with every phone call, email, and collegial discussion. Usually, when the course leads schedule their teleconferences, they present an agenda, focusing on some developmental activity for its duration. In one series of conference calls, the leads sent sample students' papers to their colleagues, and asked them to "grade" each paper prior to the call. During the call, with the Walden grading rubrics in hand, participants discussed their grading, as well as their use of the rubrics' guidelines, to reach their papers' grades. Discussion then occurred, where participants visited the Walden faculty-development site, to review posted exemplars, and compared their grading to the exemplars' results, which engendered discussion of best practices.

Whether it is honing instructors' skills with APA formatting; developing research-based practices; offering tips for providing timely, substantive, individualized feedback on students' papers; or creating a safe classroom atmosphere, faculty continue to share their expertise, experiences, and skills with one another, in a constructive, productive manner, based on performance evaluations' constructive parameters. Therefore, frequent, ongoing communication; an open forum; and a network for assessing, and developing faculty's performance are the keys to encouraging faculty development, in an active, collegial, responsive manner.

Throughout the evaluation process, faculty members have reported a willingness to improve techniques, and to share among each other their best practices in the online classroom, frequently working in a one-on-one relationship with a colleague to enhance her/his performance. In addition, the communication among instructors is that of peer-to-peer contact, rather than only coming from the administrative leadership. Such communication, and the addition of sound practices gleaned from instructors' collective experiences, has helped to bridge the cybergap inherent in the online platform, which has the potential to be misunderstood or delayed. Through the course-lead model, and the frequent collaborations among instructors, a successful communication network has led to a constructive faculty-development model. As evaluations are a means for instructors to improve their skills, so are the various ways they may communicate with their peers to support their instructional performance. The result is a faculty who work together to develop and share best practices; have a sense of empowerment, for they have created, 
and delivered, professional-development modules; and have a respected voice in shaping the educational experiences of students and colleagues alike to make a difference in distance learning at Walden University.

\section{AUTHOR INFORMATION}

Dr. Barbara Weschke is the director of the Master's in Early Reading and Literacy; Literacy and Learning in the Content Areas; and the Adolescent Reading and Literacy programs in The Richard W. Riley College of Education and Leadership of Walden University. She also has taught in these programs for the university, and is a retired department chairperson in English Language Arts in the Huntington, New York school district. She has presented at international and national professional conferences on the topics of literacy and professional development, as well conducted research on the topic of rural education and parental involvement.

Dr. Stephen Canipe is the director of the Master's of Science in Education for Math, Science, and Instructional. Design and Technology programs in The Richard W. Riley College of Education and Leadership of Walden University. He has taught at Walden University, Northern Arizona University, the University of NC-Chapel Hill, and Gaston College. In addition he has served as teacher, principal, and director in several NC school districts and was a Science Consultant with the NC Dept. of Public Instruction. Canipe has delivered numerous presentations on science, technology, assessment, and the change process to state, regional and national audiences.

\section{REFERENCES}

1. Canipe, S. L (Summer 2006). Outcomes assessment: a programmatic approach. Department Chair, Vol. 17, no. 1, 16-18.

2. Elmore, H. W. (2008, May/June). Toward objectivity in faculty evaluation. Academe, Vol. 94, Issue 3, 3840.

3. Fang, Berlin (2007, May-June). A performance-based developmental model for online faculty. Performance Improvement, 46, 17-24.

4. Koops, J. Barry, Winsor, Kim A (2005, Fall). Creating a professional learning culture through faculty evaluation. Journal of Education, 186, Issue 3, 61-70. 
NOTES 